\title{
Complement and coagulation: key triggers of COVID-19-induced multiorgan pathology
}

\author{
Berhane Ghebrehiwet ${ }^{1}$ and Ellinor I. Peerschke ${ }^{2}$ \\ 'Department of Medicine, Stony Brook University, Stony Brook, New York, USA. ${ }^{2}$ Department of Laboratory Medicine, Memorial Sloan Kettering Cancer Center, New York, New York, USA.
}

\begin{abstract}
In a stunningly short period of time, the unexpected coronavirus disease 2019 (COVID-19) pandemic, caused by severe acute respiratory syndrome coronavirus 2 (SARS-CoV-2), has turned the unprepared world topsy-turvy. Although the rapidity with which the virus struck was indeed overwhelming, scientists throughout the world have been up to the task of deciphering the mechanisms by which SARS-CoV-2 induces the multisystem and multiorgan inflammatory responses that, collectively, contribute to the high mortality rate in affected individuals. In this issue of the JCI, Skendros and Mitsios et al. is one such team who report that the complement system plays a substantial role in creating the hyperinflammation and thrombotic microangiopathy that appear to contribute to the severity of COVID-19. In support of the hypothesis that the complement system along with neutrophils and platelets contributes to COVID-19, the authors present empirical evidence showing that treatment with the complement inhibitor compstatin Cp40 inhibited the expression of tissue factor in neutrophils. These results confirm that the complement axis plays a critical role and suggest that targeted therapy using complement inhibitors is a potential therapeutic option to treat COVID-19-induced inflammation.
\end{abstract}

\section{Coronaviruses are a perpetual threat}

Coronaviruses are a group of related viruses that cause mild to severe disease in both humans and animals. However, three of the last seven coronaviruses known to date have caused much more severe and often fatal respiratory infections in humans and have been largely responsible for the deadly pneumonia outbreaks in the 21st century (1-7). In humans, coronaviruses can cause a lethal disease called severe acute respiratory syndrome (SARS), in which the lungs suffer severe injury with inability to take up oxygen, resulting in potentially deadly hypoxia (4-5). Since the first major outbreak of SARS in 2002, there have been two major coronavirus pandemics: Middle Eastern respiratory syndrome (MERS) in 2012, and the present pandemic coronavirus disease 2019 (COVID-19). Therefore, as the virus passes from species to species and mutates through either immunologic shift or immunologic drift, novel pandemic-causing strains will most certainly appear in the future. Unless an efficient vaccine or targeted therapy is generated, pandemics with coronaviruses will be a perpetual threat. Complete understanding of the pathogenesis of COVID-19 will therefore be requisite if we are to identify pharmacologic targets for the development of effective therapies in anticipation of future pandemics.

Related Article: p. 6151

Conflict of interest: The authors receive royalties from the sale of monoclonal antibodies against gC1qR clone 60.11 and clone 74.5.2. The authors hold a patent for the development of these antibodies for therapy against cancer and angioedema, respectively (US patent 8,883,153-B2, "Methods for Prevention and Treatment of Angioedema").

Copyright: @ 2020, American Society for Clinical Investigation.

Reference information: / Clin Invest. 2020;130(11):5674-5676. https://doi.org/10.1172/JCI142780.

SARS-CoV-2 infects human cells by attaching to angiotensin-converting enzyme 2 (ACE2) expressed on the epithelial cells lining the lungs, arteries, heart, kidney, and intestines (7-13). This interaction is the major determinant in tissue tropism, infectivity, and species range of the virus (7-9). Attachment of the virus then sets in motion a cascade of inflammatory events that collectively lead to severe inflammation of the lung parenchyma, impaired gas exchange with systemic release of inflammatory mediators (resulting in further inflammation), hypoxemia, and frequently, multiple organ failure. The most striking pathology in patients with COVID-19 is severe lung injury as a cumulative result of the interplay between the overactive immune and coagulation systems, which together generate conditions that exacerbate the morbidity (11-12). Increasing evidence now shows that COVID-19 pathogenesis is fueled by a maladaptive host inflammatory response that leads to excessive activation of innate pathways $(10,11)$ that generate what is known as a cytokine storm, and excessive edema leading to pulmonary fibrosis and death. The question is: what are the molecular networks that lead to the excessive activation of innate immune pathways?

Recently, Domingo et al. (12) elegantly described the pathology of COVID-19 as consisting of four feedback loops: the viral loop, the hyperinflammatory loop, the noncanonical renin-angiotensin system (RAS) loop, and the hypercoagulation loop. According to this theory, the RAS facilitates viral entry through interaction between the SARS-CoV-2 spike (S) protein and ACE2. However, in addition to ACE2, the viral S protein also binds to the serine protease TEMPRSS2, which cleaves the S protein into two functional subunits called S1 and S2 (13). The S1 binds to ACE2, whereas the S2 binds to TEMPRSS2. These two events are postulated to be responsible for fusing viralhost membranes (13). Whereas the viral loop 


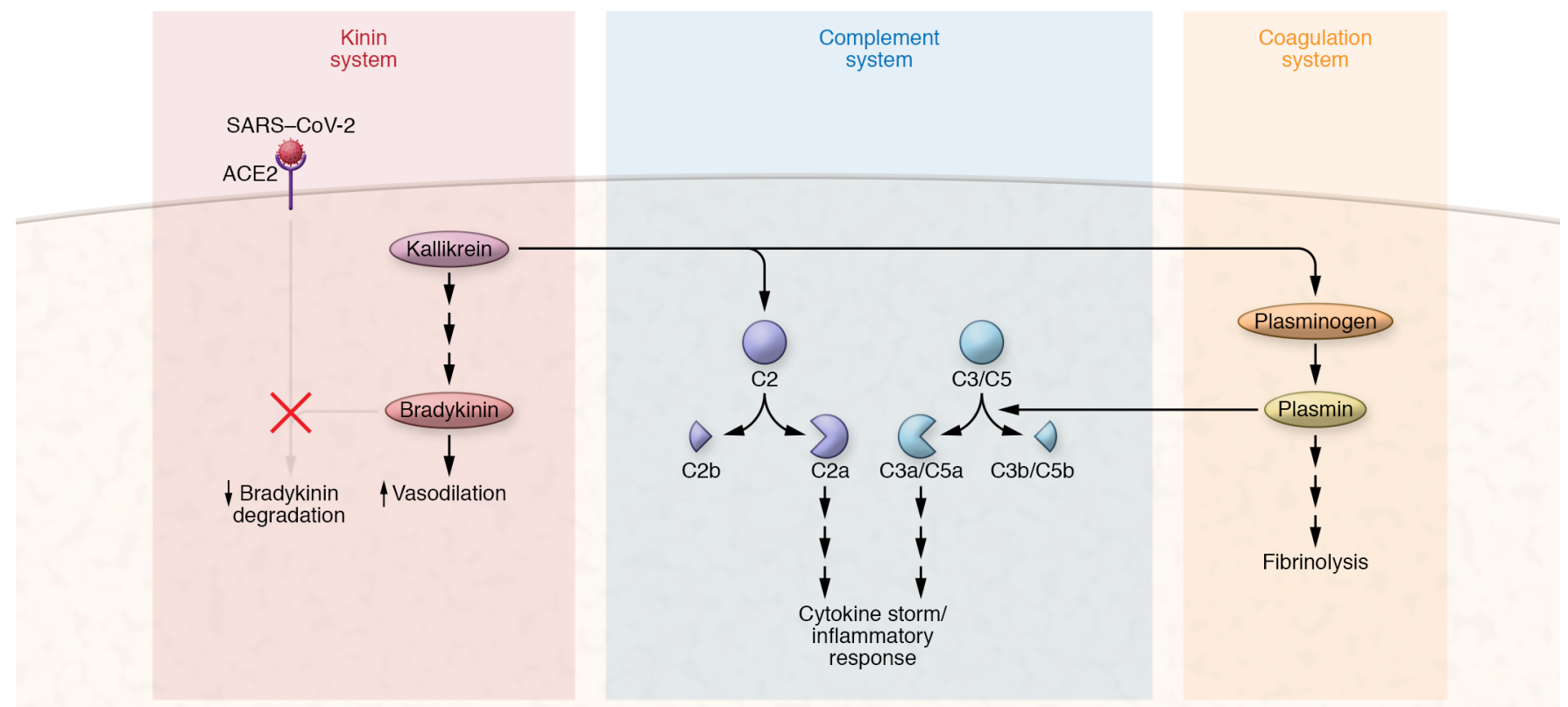

Figure 1. Cross-talk between kinin, complement, and coagulation systems. SARS-CoV-2 binding to ACE2 prevents degradation of bradykinin to dysregulate vasodilation. Kallikrein activates polypeptides to ultimately release bradykinin (BK), which results in vasodilation. Additionally, kallikrein activates the complement cascade and mediates the plasminogen cascade. Plasmin is a serine protease of plasma, which can regulate both the complement and coagulation systems. Although the major function of plasmin is to degrade fibrin clots in a process called fibrinolysis, it is also able to degrade a wide range of plasma proteins, including complement proteins, thereby generating activation fragments such as $\mathrm{C} 3 \mathrm{a}$ and $\mathrm{C} 5 \mathrm{a}$ that contribute to the cytokine storm and aggravation of the inflammatory process.

enables the virus to evade the host's innate response, resulting in uncontrolled viral replication and turning on a hyperactive immune response, the hyperinflammatory loop is largely responsible for the uncontrolled inflammatory response and recruitment of immune cells to the site of infection generating the associated cytokine storm. Finally, the hypercoagulation loop is the condition in which an uninterrupted interplay between inflammation and coagulation gives rise to an overactive inflammatory response and activation of the coagulation pathways, thereby leading to immunemediated lung injury and the signature acute respiratory distress syndrome.

Interestingly, although the primary function of ACE2 is to control the activity of ACE1 in the RAS, another important function of ACE2 is the conversion of des-Arg bradykinin (BK) into a nonfunctional pentapeptide. BK is one of the most potent vasoactive peptides known, and it is postulated to drive the edema seen in patients with COVID-19 $(14,15)$. If, as postulated, the kinin system is activated in COVID-19, then the occupancy of ACE2 by the $S$ protein would impair its ability to degrade $\mathrm{BK}$, thereby facilitating the prolonged presence of $\mathrm{BK}$ in the tissues or the blood, resulting in unregulated vascular permeability and edema (Figure 1).

\section{Cross-talk between complement and coagulation in COVID-19 pathology}

What makes the article by Skendros and Mitsios et al. (16) in this issue of the JCI timely and relevant is not only that it identifies players, such as neutrophil extracellular traps and platelets, which together with complement drive COVID-19-induced immunothrombosis, but that it also empirically confirms what had been already suspected from the very beginning of the pandemic, that the symptoms associated with COVID-19 infection were most likely brought about by a fine-tuned interplay between two of the most powerful and interactive systems of the blood, namely, the complement system (14-20) and the coagulation pathway (21). Both systems belong to the so-called triggered enzyme cascades, defined as systems where, once set in motion, the product of one reaction becomes the enzyme of the next. It is, therefore, not surprising that these systems have a common evolutionary ancestry and similar homeostatic functions with regulatory molecules - either soluble and/or membrane-associated - that are able to discriminate between healthy and altered cells. Interestingly, both systems are also simultaneously activated in response to infection or injury, and the newly generated enzymes are, in turn, able to cross-activate each other (Figure 1). For example, complement-triggered diseases clinically manifest in thrombosis, such as those seen in paroxysmal nocturnal hemoglobinuria (PNH) and atypical hemolytic uremic syndrome (aHUS), and now COVID-19. Although the functions of both systems are tightly regulated, overactivation does occur when the systems are overwhelmed by severe bacterial or viral infections, as seen in the present COVID-19 pandemic.

The complement system is comprised of three independently activated pathways and is one of the most potent arms of innate immunity. It functions to fight against infection through a variety of processes that include enhancing phagocytic cells to clear pathogenic microorganisms and/or damaged cells, destroying invading pathogens by the membrane attack complex (MAC) or C5b-9 complex, and promoting inflammation through vasoactive products generated during activation of any of the three independent pathways. However, regardless of how or which complement system pathway is activated, the end result is the formation of the MAC, which in turn is responsible for tissue damage such as that seen in patients with COVID-19. More importantly, acti- 
vation fragments such as C3a and C5a are powerful chemoattractants and can recruit monocytes and macrophages, which secrete cytokines such as IL-6, to contribute to the cytokine storm seen in infected patients. It is for this reason that therapies that target downstream events in the complement cascades have been proposed and successfully tried (17-20), including by Skendros and Mitsios et al. (16).

Data accumulated to date suggest that the coagulation system of patients with COVID-19 is highly activated, resulting in comprehensive microvascular thrombosis and the consumption of coagulation factors (19) giving rise to extensive microangiopathy and thrombocytopenia, prolongation of the prothrombin time, elevation of Ddimer, and decreased fibrinogen levels (22). In addition, diffuse thrombi are present not only in the lungs, but also in the heart, liver, and kidneys of patients who died from COVID-19 (19). Recent studies by Ramlall et al. (21) found that certain genetic disorders of the complement system (such as macular degeneration) or coagulation system (such as thrombocytopenia) are predictable risk factors for the morbidity and mortality seen in patients with SARS-CoV-2 infection, suggesting that these two pathways contribute substantially to disease severity.

The ability of COVID-19 infection to trigger simultaneous activation of multiple systems in the blood is clear. Therefore, until an effective vaccine is generated, targeted therapy against one system may inadequately silence the disease once it is triggered. Rather, a combination therapy targeting the critical steps in the various systems activated by COVID-19, including the viral attachment site (ACE2), viral structures that trigger the complement system, blood coagulation, and kinin system, as well as cytokine receptors, may provide a more efficient therapeutic option. For example, treatment of patients with the compstatin-based complement C3 inhibitor AMY-101 has already shown promise (10, 16). Similarly, blocking the signal transduction pathway of IL-6 using monoclonal antibodies such as tocilizumab and sarilumab against the IL-6 receptor (IL-6R) is also emerging as a useful strategy to prevent the cytokine storm (24). However, since entry of COVID-19 into epithelial cells depends on the interaction between the viral S protein and cell surface receptors ACE2 and TEMPRSS2, blocking viral entry may be the preferred option at the beginning of the infection. In support of this tenet is the recent trial of a clinically approved TEMPRSS2 inhibitor, which showed notable promise (13). Finally, since the kinin-kallikrein system has been strongly implicated in COVID-19 edema, therapeutic options that target BK receptors (14) as well as upstream events leading to BK generation are also likely targets that are being considered.

\section{Acknowledgments}

BG was supported in part by grants from the National Institutes of Allergy and Infectious Diseases (R01 AI 060866, R01 AI-084178, R56-AI 1223476).

Address correspondence to: Berhane Ghebrehiwet, Stony Brook University School of Medicine, Health Sciences Center, T-15; Room 060E, Stony Brook, New York, New York 11794-8161 USA. Phone: 1.631.444.2352; Email: berhane. ghebrehiwet@stonybrookmedicine.edu.

1. Fehr AR, Perlman S. Coronaviruses: an overview of their replication and pathogenesis. Methods Mol Biol. 2015;1282:1-23.

2. Masters PS. The molecular biology of coronaviruses. Adv Virus Res. 2006;66:193-292.

3. Franks TJ, et al. Lung pathology of severe acute respiratory syndrome (SARS): a study of 8 autopsy cases from Singapore. Hum Pathol. 2003;34(8):743-748.

4. Hwang DM, et al. Pulmonary pathology of severe acute respiratory syndrome in Toronto. Pathol. 2005;18(1):1-10.

5 . Nicholls JM, et al. Lung pathology of fatal severe acute respiratory syndrome. Lancet. 2003;361(9371):1773-1778.

6. Cui J, Li F, Shi ZL. Origin and evolution of coronaviruses. Nat Rev Microbiol. 2019;17(3):181-192.

7. Cruz JL, et al. Alphacoronavirus protein 7 modulates host immune response. J Virol. 2013;87(17):9754-9767.

8. Li F, Li W, Farzan M, Harrison SC. Structure of SARS coronavirus spike protein receptor-binding domain complexed with receptor. Science. 2005;309(5742):1864-1868.
9. Chan KH, Tsang NC, Guan Y, Yuen KY, Peiris JS. Lung pathology of fatal severe acute respiratory syndrome. Lancet. 2003;361(9371):1773-1778.

10. Mastaglio S, et al. The first case of COVID-19 treated with the complement C3 inhibitor AMY101. Clin Immunol. In press.

11. Gralinski LE, et al. Mechanisms of severe acute respiratory syndrome coronavirus-induced acute lung injury. mBio. https://doi:10.1128/ mBio.00271-13.

12. Domingo $P$, et al. The four horsemen of a viral apocalypse: The pathogenesis of SARS-CoV-2 infection (COVID-19). eBioMed. https://doi: https://doi.org/10.1016/j.ebiom.2020.102887.

13. Hoffmann M, et al. SARS-CoV-2 cell entry depends on ACE2 and TMPRSS2 and is blocked by a clinically proven protease inhibitor. Cell. 2020;181:272-280.

14. Van de Veerdonk, et al. Kallikrein-kinin blockade in patients with COVID-10 prevent acute respiratory distress syndrome. eLife. https://doi. org/10.7554/eLife.57555.

15. Kaplan AP, Ghebrehiwet B. The plasma bradykinin-forming pathways and its interrelationships with complement. Molec Immunol. 2010;47(13):2161-2169.

16. Skendros P, Mitsios A, et al. Complement and tissue factor-enriched neutrophil extracellular traps are key drivers in COVID-19 immunothrombosis. JClin Invest. 2020;130(11):6151-6157.

17. Mastaglio S, et al. The first case of COVID-19 treated with the complement C3 inhibitor AMY101. Clin Immunol. 2020;215:108450.

18. Stahel PF, Barnum SR. Complement inhibition in coronavirus diseases (COVID)-19: A neglected therapeutic option. Front Immunol. Published online July 7, 2020. https://doi.org/10.3389/ fimmu.2020.01661.

19. Noris M, Benigni A, Remuzzi G. The case of complement activation in COVID-19 multiorgan impact. Kidney International. 2020;98(2):314-322.

20. Risitano AM, Mastellos DC, et al. Complement as a target in COVID-19. Nat Rev Immunol. 2020;20(6):343-344.

21. Ramlall V, et al. Immune complement and coagulation dysfunction in adverse outcomes of SARS-CoV-2 infection. Nat Med. Published online August 3, 2020. https://doi.org/10.1038/ s41591-020-1021-2.

22. Tang N, Li D, Wang X, Sun Z. Abnormal coagulation parameters are associated with poor prognosis in patients with novel coronavirus pneumonia. J Thromb Haemost. 2020;18(4):844-847.

23. Gralinski LE, et al. Complement activation contributes to severe acute respiratory syndrome coronavirus pathologies. mBio. Published online October 9, 2018. https://doi.org/10.1128/ mbio.01753-18.

24. Luo P, et al. Tocilizumab treatment in COVID19: a single center experience. JMed Virol. 2020;92(7):814-818. 